\title{
(C) OPEN ACCESS \\ British Neurotoxin Network \\ recommendations for managing cervical dystonia in patients with a poor response to botulinum toxin
}

\author{
Marie-Helene Marion, ${ }^{1,2}$ Miles Humberstone, ${ }^{3,4}$ Richard Grunewald, $^{5}$ \\ Sunil Wimalaratna ${ }^{6}$
}

\begin{abstract}
'London BTX Centre, London, UK

${ }^{2}$ British Neurotoxin Network, Guildford, UK

${ }^{3}$ Department of Neurology, Nottingham University Hospitals, Nottingham, UK

${ }^{4}$ Department of Neurology, United Lincolnshire Hospitals, Lincoln, UK

${ }^{5}$ Department of Neurology, Sheffield Teaching Hospitals NHS FT, Sheffield, UK ${ }^{6}$ Department of Neurology, Kettering General Hospital NHS Foundation Trust, Kettering, UK
\end{abstract}

\section{Correspondence to}

Dr Marie-Helene Marion, London BTX Centre, 9a Wilbraham Place Practice, London SW1X 9AE, UK;

mariehelenemarion@gmail.com

Accepted 17 February 2016 Published Online First 14 March 2016
CrossMark

To cite: Marion M-H, Humberstone $M$, Grunewald $\mathrm{R}$, et al. Pract Neurol 2016;16:288-295.

\begin{abstract}
Botulinum toxin (BoNT) injections are an effective treatment for cervical dystonia. Approximately

$20 \%$ of patients eventually stop BoNT treatment, mostly because of treatment failure. These

recommendations review the different

therapeutic interventions for optimising the treatment in secondary poor responder patients. Immunoresistance has become less common over the years, but the diagnosis has to be addressed with a frontalis test or an Extensor Digitorum Brevis test. In case of immunoresistance to BoNT$A$, we discuss the place the different therapeutic options (BoNT-A holidays, BoNT-B injections, alternative BoNT-A injections, deep brain stimulation). When poor responders are not immunoresistant, they benefit from reviewing (1) injections technique with electromyography or ultrasound guidance, (2) muscles selection and (3) dose of BoNT. In addition, in both scenarios, a holistic approach including drug treatment, retraining and psychological support is valuable in the management of these complex and severe cervical dystonia.
\end{abstract}

Cervical dystonia impairs quality of life and leads to social and occupational disability: botulinum toxin injections are an effective treatment. ${ }^{1}$ About 20\% of patients with cervical dystonia eventually stop botulinum toxin treatment, mostly after treatment failure. ${ }^{2}$ Spontaneous remission, improvement after long-term treatment or difficulty accessing the treatment (eg, living in remote areas) can also lead to stopping the treatment.

These recommendations review the therapeutic interventions to optimise the treatment of patients with a poor response. We provide references that give the evidence base to guide treatment; the non-referenced advice should be taken as the authors' opinion, based on experience. The British Neurotoxin Network members, at the annual meeting in Oxford in September 2015, have endorsed these guidelines.

\section{DEFINITIONS AND TERMINOLOGY}

- Poor responders (treatment failures) are those who, subjectively or from the doctor's observation, do not achieve adequate symptom relief (primary or secondary non-responders) or who develop intolerable adverse effects from treatment (dysphagia, neck extensors weakness). ${ }^{3}$

- Primary non-responders are those in whom botulinum toxin treatment has never helped.

- Secondary non-responders are those who fail to respond following previously successful treatment to botulinum toxin. A widely accepted definition of secondary non-response is "an unsatisfactory therapeutic response to two successive injection cycles, where the patient has previously received a minimum of two successful treatment cycles". ${ }^{4}$

- We use UK brand names of botulinum toxin preparations to facilitate the use of these guidelines to UK injectors. Table 1 shows the different preparations of botulinum toxin type A (botulinum toxin-A) and botulinum toxin type B (botulinum toxin-B) with their brand names and their US generic names, as requested by the FDA to emphasise the difference between these preparations. ${ }^{5}$

\section{CHALLENGES WHEN TREATING CERVICAL DYSTONIA WITH BOTULINUM TOXIN}

Botulinum toxin treatment has to be customised to each individual patient. The injector's skill comprises a combination 
Table 1 Different preparations of botulinum toxin

\begin{tabular}{llll}
\hline US generic name & $\begin{array}{l}\text { Brand } \\
\text { name }\end{array}$ & Manufacturer & $\begin{array}{l}\text { Packaging } \\
\text { Units/phial }\end{array}$ \\
\hline OnabotulinumtoxinA & Botox & Allergan & $50,100,200$ \\
AbobotulinumtoxinA & Dysport & Ipsen & 300,500 \\
IncobotulinumtoxinA & Xeomin & $\begin{array}{l}\text { Merz } \\
\text { Pharmaceuticals }\end{array}$ & 50,100 \\
RimabotulinumtoxinB & NeuroBloc & Eisai Ltd & 2500,5000 , or \\
& & & 10000
\end{tabular}

These four preparations are available and licensed in the UK for the treatment of cervical dystonia. The units between these different preparations are not equivalent, and the clinician must be vigilant when switching from one preparation to another. As an indication, 1 unit Botox $=1$ unit Xeomin $=3$ units Dysport=50 units Neurobloc for cervical dystonia.

of postural analysis, identification of offending muscle groups, optimisation of dosage, dilution ratios, interval between injections, injection technique and, when necessary, targeting the muscles guided by electromyography (EMG) or ultrasound. The optimal treatment will relieve the patient's disability with minimal or no adverse effects.

\section{REASONS FOR TREATMENT FAILURES}

There have been several studies looking at large cohorts of patients with cervical dystonia and the reasons for treatment failures. ${ }^{4}{ }^{6-9}$ Most poor responses relate to suboptimal treatment and a minority to immunoresistance from developing neutralising antibodies. Secondary non-response (to two consecutive injections cycles) may also relate to underlying disease progression. ${ }^{10}$

Adverse effects can limit optimal treatment, in particular, dysphagia, in patients receiving bilateral injections of sternocleidomastoid or longus colli, when there are pre-existing swallowing difficulties ${ }^{11}$ or in craniocervical dystonia when injections target both neck and tongue muscles.

Some patients perceive a poor response through unrealistic expectations, depressed mood or when they compare their current response to the initial response, which may have had the most noticeable benefit.

\section{IMMUNORESISTANCE}

\section{Prevalence of immunoresistance in non-responders}

Recent studies show relatively low rates of neutralising antibodies with using botulinum toxin- $\mathrm{A},{ }^{12}$ and that these antibodies are not closely associated with treatment failure. Rates of treatment failure associated with neutralising antibodies with Dysport range from $0 \%{ }^{10}$ to $2.5 \%,{ }^{4}$ with Xeomin range from $0 \%{ }^{13}$ to $1 \%$ in patients who had previously received other botulinum toxin-A products, ${ }^{14}$ and with Botox from $0 \%{ }^{13}$ to $1.2 \% .^{16}$

Earlier reports of a higher prevalence neutralising antibodies $(17-23 \%)$ may be because the original toxin formulation had a higher protein concentration. ${ }^{15} 17$

Botulinum toxin-B was approved to treat cervical dystonia in 2001 in the UK and may be an alternative for patients resistant to botulinum toxin-A. Botulinum toxin- $\mathrm{B}$ is effective and safe in the long-term treatment of cervical dystonia, ${ }^{18}$ but its higher immunogenicity-in particular, in patients already resistant to botulinum toxin-A-may limit its use as a therapeutic alternative. ${ }^{19-20}$

Neutralising antibody titres to botulinum toxin-A decline after stopping botulinum toxin therapy in resistant patients. ${ }^{21}$ The rate of decline varies considerably between individuals, and it can take up to 4 years after stopping treatment before the antibody titres become undetectable. ${ }^{21}$ Antibody titres also decline in resistant patients when treated with Xeomin. ${ }^{21}$ However, in one report a patient pretreated with Dysport developed a secondary antibodyinduced treatment failure under therapy with Xeomin. ${ }^{22}$ Therefore, we await confirmation of the efficacy of Xeomin in resistant patients.

\section{Early diagnosis of immunoresistance}

Botulinum toxin-A resistance may occur very early in the course of treatment, usually within $2-3$ years after starting treatment; the response usually fails gradually, starting with reduced duration of clinical effect, followed by significantly reduced maximal effect. ${ }^{7}$ Patients with cervical dystonia should therefore be carefully monitored with scoring of the treatment effect in order to detect immunoresistance early, thereby avoiding complete therapy failure with high titres of neutralising antibodies. ${ }^{23}$ Objective tests include unilateral injection of the frontalis muscle or injection of the extensor digitorum brevis of the foot with botulinum toxin-A. The frontalis test is the most frequently used method. ${ }^{24}$ Any evidence of paralysis (frontalis or extensor digitorum brevis test) excludes immunoresistance as the cause of treatment failure.

\section{Frontalis test}

Two injections of 30 units Dysport or 10 units Botox $^{25}$ or Xeomin are given to the forehead about $3 \mathrm{~cm}$ above the lateral and medial canthus of one eye (figure 1). The response is assessed 2-4 weeks later by asking the patient to raise their eyebrows: an asymmetric response indicates that botulinum toxin has been effective. ${ }^{25}$ To avoid equivocal results (especially in cases of partial resistance), it is valuable to compare photographs or videos before and after injection. If the frontalis test is equivocal, the clinician can use a higher dose of botulinum toxin- $\mathrm{A}$ or perform the extensor digitorum brevis test to give quantified data.

Extensor digitorum brevis test using EMG (electrical stimulation)

For the extensor digitorum brevis test, 100 units Dysport or 20 units Botox are injected into the 
A

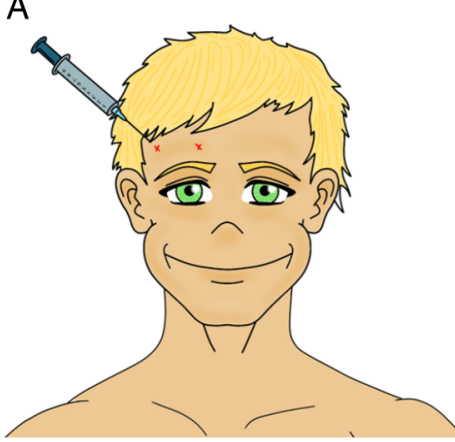

B

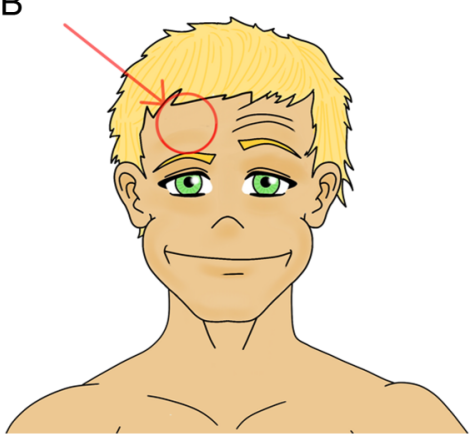

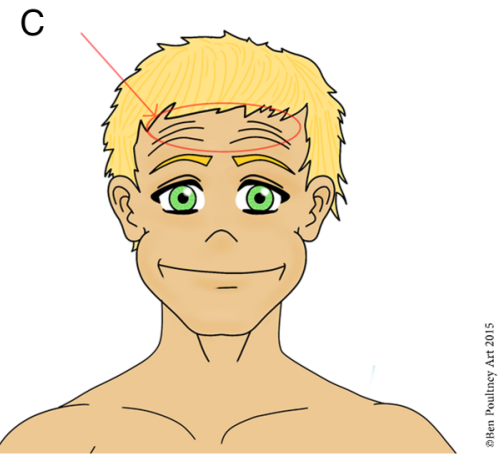

Figure 1 (A) Frontalis test: unilateral injection of the frontalis muscle with botulinum toxin (BoNT). (B) Patient non-resistant to BoNT: paralysis of the frontalis muscle and incapacity to raise the eyebrow on the side of the injection. (C) Patient resistant to BoNT: absence of paralysis of the frontalis muscle on the side of the injection and symmetrical elevation of eyebrows.

muscle on one side. EMG measures the compound muscle action potential (CMAP) of the extensor digitorum brevis using electrical stimulation of the peroneal nerve at baseline and 2 weeks after botulinum toxin-A injection. ${ }^{26}$ If the toxin is biologically active, the CMAP should fall by $>50 \%$ of the baseline values, usually with evidence of atrophy and weakness. A fall in CMAP of $<20 \%$ suggests the presence of neutralising antibodies; a $20-50 \%$ decrease is equivocal.

\section{MEDICAL AND SURGICAL TREATMENT OF CERVICAL DYSTONIA}

There are only a few studies on drug treatment in dystonia. $^{27} 28 \mathrm{~A}$ comparison ${ }^{28}$ of botulinum toxin-A (mean dose 292 units Dysport) versus trihexyphenidyl (mean dose $16.25 \mathrm{mg}$ ) in cervical dystonia favoured botulinum toxin-A. Recent experimental evidence suggests that anticholinergic drugs can help dystonia ${ }^{29}$ but patient's increased risk of dementia from longterm anticholinergic drugs is a concern. ${ }^{30}$
Surgical treatment of cervical dystonia has evolved from peripheral denervation to pallidal deep brain stimulation (GPi-DBS). ${ }^{31}$ A recent comparative study between the two procedures favoured GPi-DBS, which gave greater benefit but with potentially more severe complications. ${ }^{32}$ Bradykinesia, leg stiffness and freezing of gait can develop with high-frequency stimulation GPi-DBS in cervical dystonia. ${ }^{33}$

\section{RECOMMENDATIONS FOR MANAGING PRIMARY POOR RESPONDERS AND SECONDARY NON-RESPONDERS}

A European survey of management of secondary nonresponse to botulinum toxin-A in cervical dystonia found that the physicians' most common practice was to optimise physiotherapy (98\%), to increase the dose of botulinum toxin-A (89\%), to target new muscles with the treatment (91\%) and to use EMG (86\%). Also, $64 \%$ of physicians considered switching to botulinum toxin-B and $73 \%$ considered switching to DBS; $18 \%$ considered peripheral denervation. ${ }^{24}$

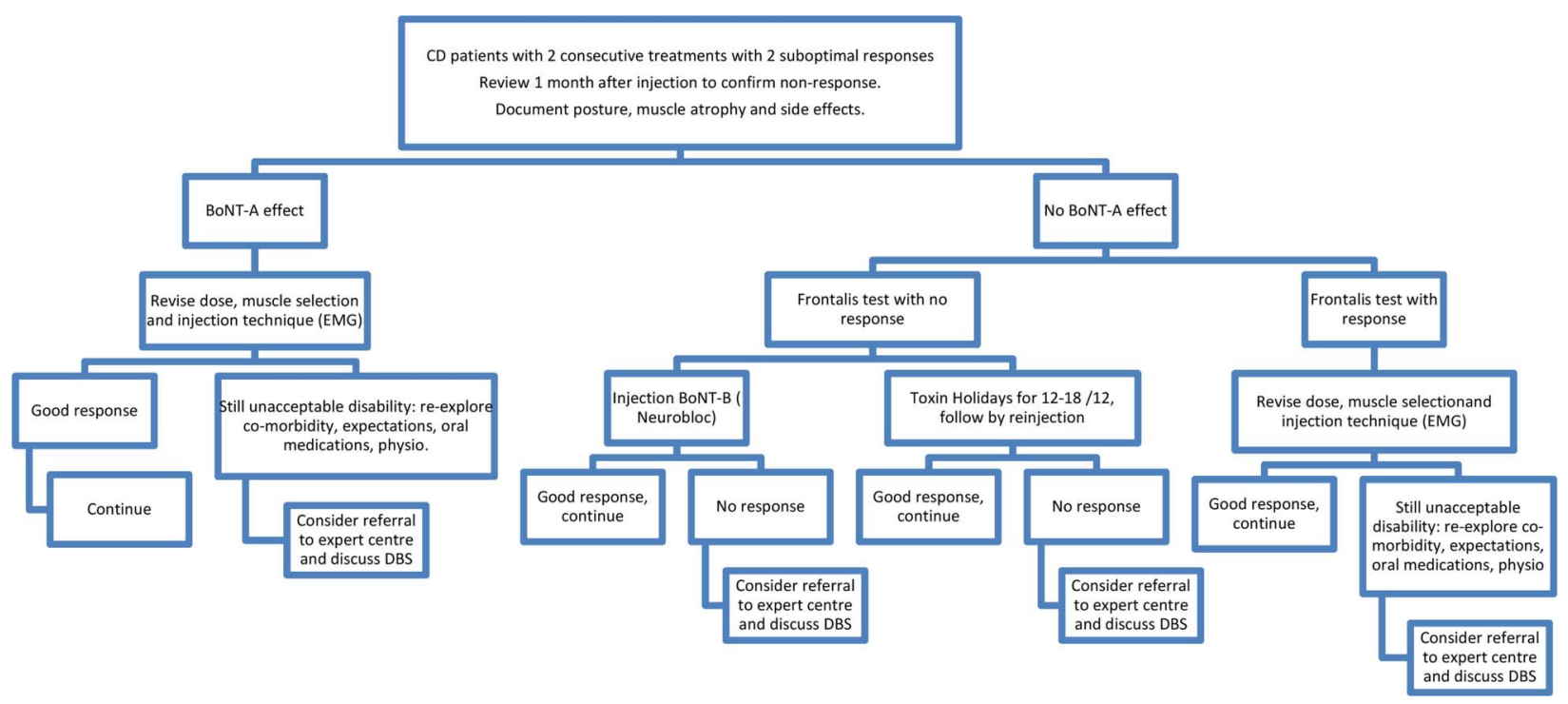

Figure 2 Algorithm for the management of patients with cervical dystonia who have a poor response to botulinum toxin treatment. BoNT, botulinum toxin; CD, cervical dystonia; DBS, deep brain stimulation; EMG, electromyography. 


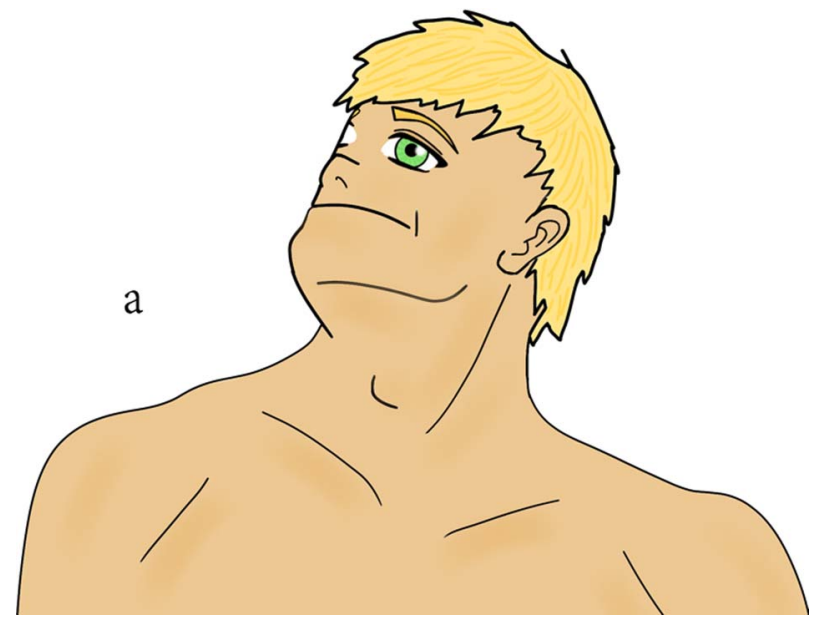

Figure 3 Mixed dystonic head posture: rotation of the head to the right, extension and tilt to the left with elevation of right shoulder. The muscles involved are left sternocleidomastoid, right splenius and right levator scapulae.

There are no published guidelines for managing patients with cervical dystonia who are poor responders. From the above evidence and from our UK clinical experience, we propose an algorithm in figure 2 to support the UK injectors.

\section{Document the poor clinical response}

- Assess the patient before injection and preferably 1 month after the injections using either a rating scale or goniometric measurement of head posture and/or video clips of the patient sitting, standing, walking; sitting eyes opened and closed; and performing active movement of head.

- Document wasting of injected muscles, in particular, the sternocleidomastoid.

- Ask patients specifically about dysphagia (fluid or solids) before and after injections. Adverse effects may limit dose but are clear evidence of ongoing sensitivity to botulinum toxin.

- If botulinum toxin-A appears effective (muscle atrophy, side effects), revise the injection protocol (muscle selection, doses, injection technique).

\section{Review the muscle selection}

- Patients often have a mixed pattern of cervical dystonia (figure 3) (typically a combination of extension, rotation

Table 2 Posterior muscle layers of the neck

\section{Four layers}

\begin{tabular}{ll}
\hline $\begin{array}{l}\text { Superficial layer; } \\
\text { oblique running posterior to anterior } \\
\text { Layer of muscles, oblique anterior to } \\
\text { posterior }\end{array}$ & Trapezius \\
Layer of muscles with vertical orientation & $\begin{array}{l}\text { Splenius capitis and cervicis } \\
\text { Levator scapulae } \\
\end{array}$ \\
& $\begin{array}{l}\text { Semispinalis capitis } \\
\text { Longissimus capitis and } \\
\text { cervicis }\end{array}$ \\
Deep layer & Suboccipital muscles \\
& Multifidi muscles \\
\hline
\end{tabular}

Table 3 The main group of muscles involved in dystonic posture

\begin{tabular}{|c|c|}
\hline Action & Muscles groups \\
\hline Torticollis & $\begin{array}{l}\text { Contralateral sternocleidomastoid } \\
\text { Contralateral trapezius } \\
\text { Ipsilateral splenius capitis } \\
\text { Ipsilateral levator scapulae }\end{array}$ \\
\hline Laterocollis & $\begin{array}{l}\text { Ipsilateral sternocleidomastoid } \\
\text { Ipsilateral splenius capitis } \\
\text { Ipsilateral levator scapulae } \\
\text { Ipsilateral trapezius }\end{array}$ \\
\hline Retrocollis & $\begin{array}{l}\text { Bilateral splenius } \\
\text { Bilateral semispinalis }\end{array}$ \\
\hline Antecollis & $\begin{array}{l}\text { Bilateral sternocleidomastoid } \\
\text { Bilateral longus colli } \\
\text { Bilateral scalenus }\end{array}$ \\
\hline
\end{tabular}

This table guides the clinical examination to certain muscles.

and tilt to different degrees) rather than pure rotation, tilt, flexion or extension; this makes a dystonic movement very different from a normal purposeful head/neck movement (tables 2-4). More rarely there is lateral and sagittal shift of the head and neck. Often the scapular muscles are also involved in the abnormal posture of head and neck.

- The patient may adopt a compensatory posture in opposition to the dystonic spasm, which can be misleading for the clinician. Therefore, analysing the dystonic posture can be difficult; in addition, there can be tremor and myoclonus superimposed on the dystonic spasms.

- Be sure to observe the patient at their worst. Abnormal postures can be variable and task-specific (eg, walking, running, drinking, writing, answering the telephone). Video clips of daily activities can help.

- Examine the shoulder muscles, looking for elevation of one shoulder, comparing the position of the scapulae and assessing hypertrophy of the scapular portion of the levator scapulae. Be aware (figure 4) of the synergy between contralateral sternocleidomastoid and contralateral trapezius, and between ipsilateral levator scapulae and ipsilateral splenius for rotation (table 3). Shoulder elevation on the side of rotation therefore suggests dystonic levator scapulae, and shoulder elevation on the opposite side of rotation suggests dystonic trapezius (figure 4).

Table 4 Muscles involved in lateral shift of the head, also known as 'Bali dancer's posture'

\begin{tabular}{lll}
\hline & $\begin{array}{l}\text { Tilt of head to one } \\
\text { side }\end{array}$ & $\begin{array}{l}\text { Tilt of the neck to the } \\
\text { contralateral side }\end{array}$ \\
\hline $\begin{array}{l}\text { Head attachment } \\
\text { muscles }\end{array}$ & $\begin{array}{l}\text { Ipsilateral } \\
\text { sternocleidomastoid } \\
\text { Ipsilateral splenius } \\
\text { capitis } \\
\text { Ipsilateral trapezius } \\
\text { Ipsilateral semispinalis } \\
\text { capitis }\end{array}$ & \\
Cervical spine & & $\begin{array}{l}\text { Contralateral levator } \\
\text { attachment muscles }\end{array}$ \\
\hline
\end{tabular}



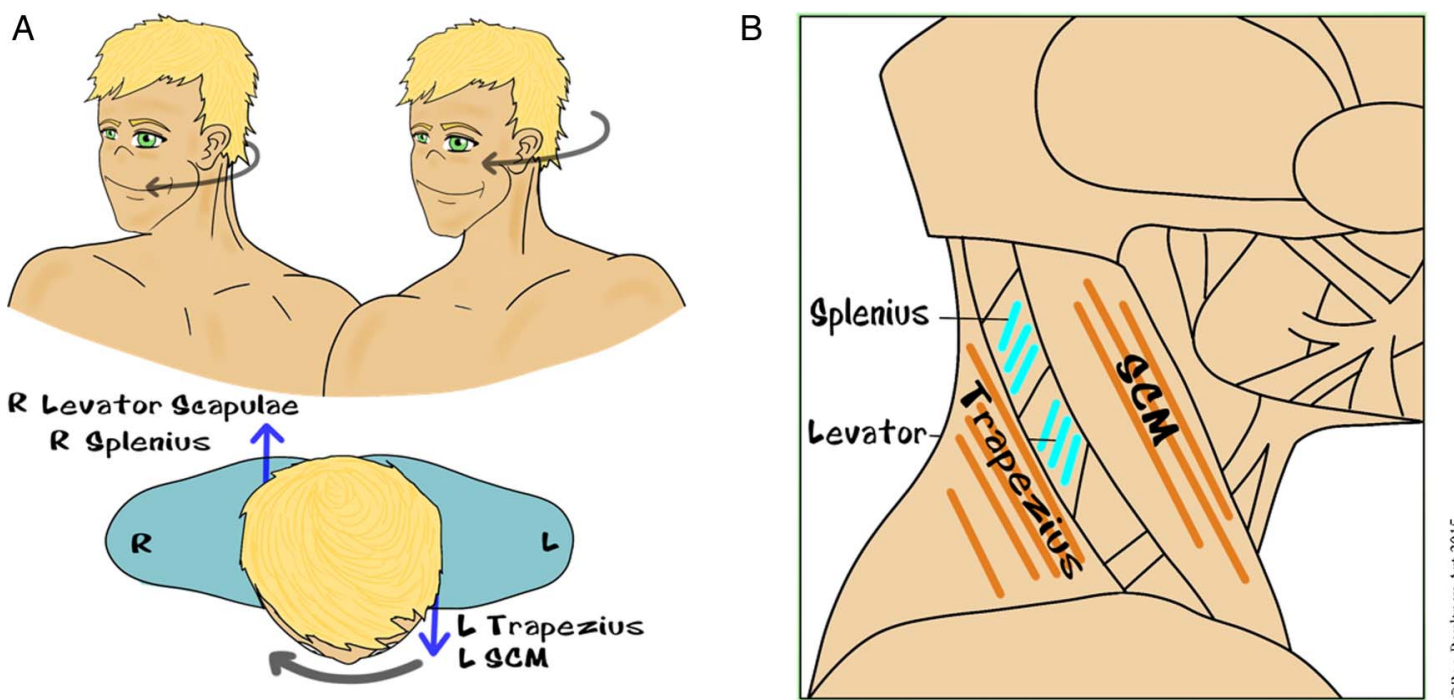

Figure 4 (A) Clinical selection of scapular muscles depending on the side of the elevation of the shoulder in a patient with dystonic head rotation. (B) Identical muscle fibre orientation explains the synergy of action in the rotation of the head between sternocleidomastoid and trapezius and between splenius and levator scapulae.

- In laterocollis, after a few injections the patient may develop a horizontal translation of the head with the shift of the neck towards one side and the head towards the opposite side (figure 5, table 4). This lateral shift of the head is very similar to the so-called 'head slide' movement seen in Balinese dance.

- In cases of posterior sagittal shift (double-chin posture; figure 5), we recommend injecting the longus colli and the supra-hyoid muscles. In cases of anterior sagittal shift (goose-neck posture; figure 5), we recommend injecting both bilateral sternocleidomastoid and bilateral splenius capiti. ${ }^{34}$

- In antecollis (figure 6), it is often not sufficient to inject both sternocleidomastoids. In cases of double-chin posture (table 5), clinicians should consider additional injection of longus colli and the supra-hyoid muscles. Be aware of the risk of dysphagia with these injections.

- In cases of severe retrocollis (figure 6), it is difficult, in practice, to distinguish the extension of the cervical spine (hyperlordosis) from the extension of the head on cervical spine. In that case, it is more important to choose a high dose of botulinum toxin (see section 'Review dosage') and an appropriate needle (see section

Table 5 Muscles involved in sagittal shift of the head

\begin{tabular}{lll}
\hline & $\begin{array}{l}\text { Anterior sagittal } \\
\text { shift } \\
\text { 'Goose-neck posture' }\end{array}$ & $\begin{array}{l}\text { Posterior sagittal } \\
\text { shift } \\
\text { 'Double-chin posture' }\end{array}$ \\
\hline Head attachment & Bilateral & $\begin{array}{l}\text { Bilateral longus colli } \\
\text { muscles }\end{array}$ \\
& sternocleidomastoid & $\begin{array}{l}\text { Bilateral } \\
\text { sternocleidomastoid } \\
\text { Supra-hyoid muscles }\end{array}$ \\
\hline
\end{tabular}

The anterior sagittal shift of the head, also called 'goose-neck posture', can be the first step of the swing of the head backward with extension of head on cervical spine and cervical spine on thoracic spine. The posterior sagittal shift of the head also called 'double-chin posture', can be the first step of the swing of the head forward with flexion of head on cervical spine and cervical spine on thoracic spine.
'Review injection technique') rather than to try to distinguish each muscle of the posterior cervical region during the injections as all the posterior cervical muscles can be involved in the extension (table 2).

- The suboccipital muscles may be involved in some abnormal postures of the head such as rotation, extension or tilt of head on the cervical spine. However, injecting these muscles may carry risk due to the proximity of the vertebral artery.

\section{Review dosage}

- The dose can be increased according to the clinical effect of previous injections.

- The dose injected into sternocleidomastoid is limited by the risk of dysphagia; the recommended dose is 50100 units of Botox/Xeomin in two sites (SPC data) and 150 units of Dysport. In our experience, the splenius requires a much larger dose than the sternocleidomastoid.

- The total dose can be increased up to 1000 units Dysport and 300 units Botox/Xeomin (SPC data). Patients with retrocollis often need large doses.

\section{Review injection technique}

- The needle used to inject the posterior cervical muscles from the second and third muscles layers (table 2) needs to be long enough to reach these muscles; we recommend using long blue needles $(23 \mathrm{~g} / 31.5 \mathrm{~mm})$ or grey needles $(27 \mathrm{~g} / 40 \mathrm{~mm})$.

- Consider EMG-guided or ultrasound-guided injections if there is suspected deep muscle involvement or, in patients with a thick neck, to help target the dystonic muscles.

\section{Have a holistic therapeutic approach}

- Review the drug treatment and consider a retraining programme (physiotherapy, mindfulness, mental imagery), 
A

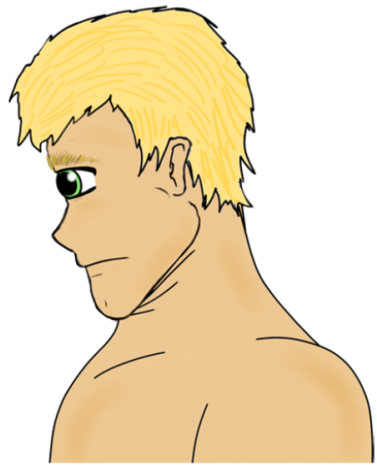

B

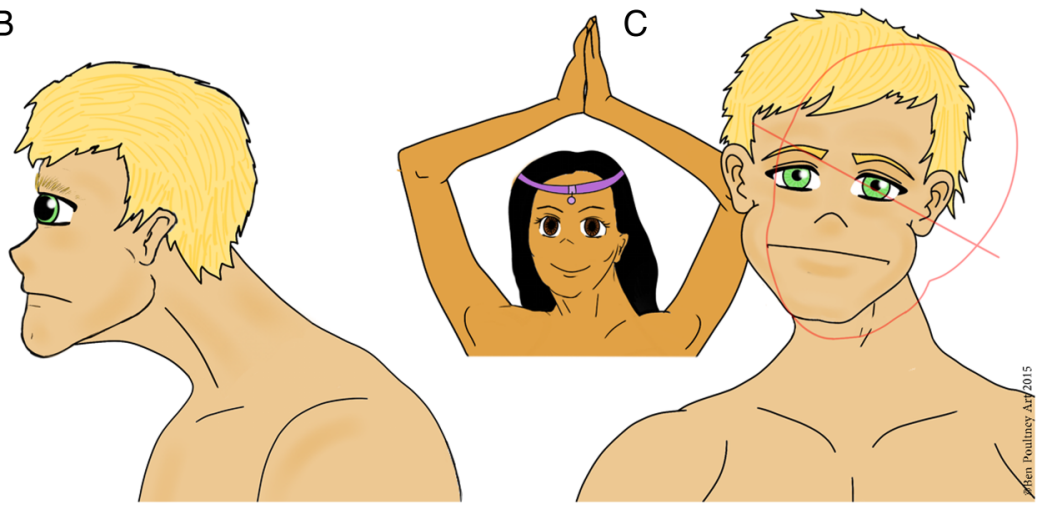

Figure 5 (A) Posterior shift of the head or double chin. (B) Anterior shift of the head or goose neck. (C) Lateral shift of the head: the contralateral shift of the neck to the right allows the patient to have a horizontal gaze despite a tilt of his head to the left.

taking into account stressful events, which can make the dystonia transiently worst.

- Anticholinergic drugs, in particular, trihexyphenidyl, may help the spasms and are usually well tolerated if introduced very gradually over few months. They are used up to a typical dose of $6 \mathrm{mg} /$ day according to response and side effect profile, although some experienced clinicians may choose use higher doses. Anticholinergic drugs particularly help poor responding patients, and their prescription should be reviewed annually.

- Benzodiazepines, in particular, clonazepam, may help those with additional tremor or myoclonus. These should also be introduced very gradually, aiming for a maximum dose of $1.5 \mathrm{mg} /$ day. The patient should be warned about habituation and tolerance.

\section{Consider immunoresistance}

If there is evidence of loss of botulinum toxin-A efficacy (poor clinical response, absence of muscle wasting and absence of side effects), undertake a frontalis test or an extensor digitorum brevis test to diagnose immunoresistance; this should be done early to avoid further increase of antibodies titres.

For patients with immunoresistance to botulinum toxin $\mathrm{A}$, there are two initial options:

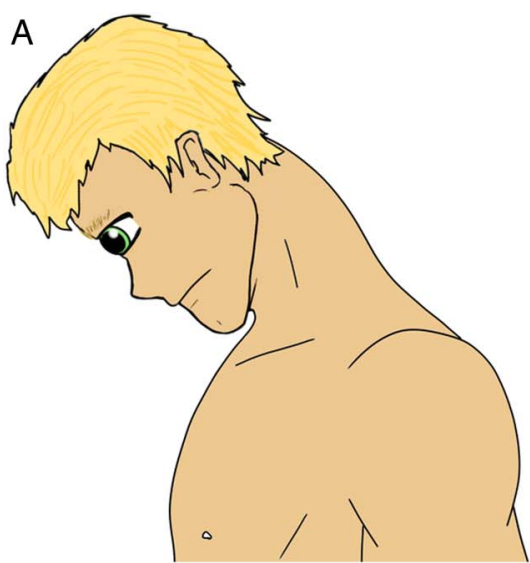

1. Use NeuroBloc (botulinum toxin-B) injections.

2. Suspend injections for $12-18$ months, reviewing the patient regularly. If they have impaired quality of life, start a new cycle of injections using an alternative botulinum toxin-A.

If there is no clinical response or if patients develop further immunoresistance to botulinum toxin- $\mathrm{B}$, consider DBS.

\section{Referral to expert centre for second opinion}

We advise referral to an expert centre in the following circumstances:

- if the severity of dystonia is not controlled despite reviewing injection protocol;

- when there is a very complex dystonic posture, such as antecollis, horizontal or sagittal shift of the head requiring injection of deep muscles such as longus colli, scalenus or the suboccipital muscles;

- when large doses of toxin are required (up to 400 units Botox-or Xeomin and 1500 units of Dysport), for severe dystonic spasms, involving more than three large muscles (splenius, semispinalis, levator scapulae, trapezius);

- when DBS is indicated (patient has a poor quality of life despite optimal treatment or in cases of immunoresistance).

B

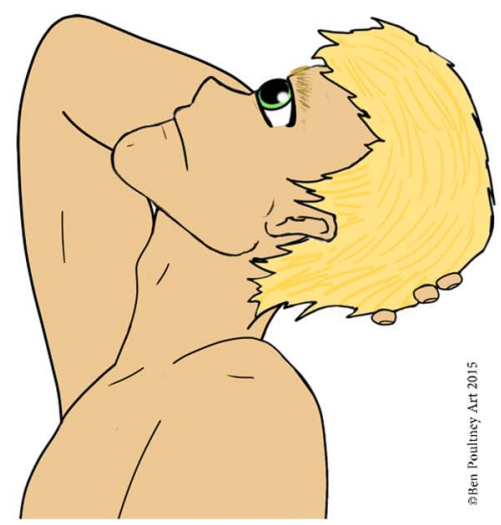

Figure 6 (A) Antecollis posture with flexion of head and neck. (B) Retrocollis posture with extension of head and neck. 


\section{CONCLUSIONS}

It is important to be alert to a patient's complaints of poor response to botulinum toxin treatment despite the difficulties of working in often overloaded and routine clinics with short timeslots for treatment.

In such cases, it is important to be proactive without delay:

- Review injection technique (with EMG guidance), dose and selection of muscles to improve the efficacy of the injections.

- Look for immunoresistance by frontalis or extensor digitorum brevis test.

- Refer to an expert centre for challenging and complex cases for advice and to discuss surgical options (such as DBS).

Secondary non-response is not necessarily a failure but is a therapeutic challenge for which there may be specific answers.

\section{Key points}

- Immunological resistance to botulinum toxin type A is becoming rare, but early diagnosis is important.

- Most patients with cervical dystonia who are nonresponders require review of the clinical analysis of the dystonic posture.

- Do not forget to examine the shoulders as levator scapulae and trapezius are powerful muscles!

- Severe cervical dystonia requires a high dose range of botulinum toxin type $\mathrm{A}$.

- Think beyond botulinum toxin type $A$ injections: a holistic approach with physical retraining is an important part of the treatment.

Acknowledgements The authors thank Mr Ben Poultney for the illustrations of this article, drawn from pictures of patients with dystonia. They are also grateful to the BNN participants, who have reviewed these guidelines at the annual meeting in Oxford in September 2015.

Competing interests $\mathrm{M}-\mathrm{HM}$ reports grants given to the $\mathrm{BNN}$ from Allergan, Merz and Ipsen, in equal proportion outside the submitted work; and M-HM has received honorarium from Merz Pharma to speak at meetings. MH has received honoraria from Merz Pharma and has accepted sponsorship to attend educational meetings from Allergan, Ipsen, Merz and Elan.

Provenance and peer review Commissioned; externally peer reviewed. This paper was reviewed by Thomas Kimber, Adelaide, Australia

Open Access This is an Open Access article distributed in accordance with the Creative Commons Attribution Non Commercial (CC BY-NC 4.0) license, which permits others to distribute, remix, adapt, build upon this work noncommercially, and license their derivative works on different terms, provided the original work is properly cited and the use is non-commercial. See: http://creativecommons.org/licenses/by$\mathrm{nc} / 4.0 /$

\section{REFERENCES}

1 Albanese A, Asmus F, Bhatia KP, et al. EFNS guidelines on diagnosis and treatment of primary dystonias. Eur J Neurol 2011;18:5-18.
2 Comella CL, Thompson PD. Treatment of cervical dystonia with botulinum toxins. Eur J Neurol 2006;13(Suppl 1):16-20.

3 Evidente VGH, Pappert EJ. Botulinum toxin therapy for cervical dystonia: the science of dosing. Tremor Other Hyperkinet Mov (N Y) 2014;4:273.

4 Kessler KR, Skutta M, Benecke R, et al. Long-term treatment of cervical dystonia with botulinum toxin A: efficacy, safety, and antibody frequency. German Dystonia Study Group. J Neurol 1999;246:265-74.

5 Albanese A. Terminology for preparations of botulinum neurotoxins: what a difference a name makes. JAMA 2011;305:89-90.

6 Wissel J, Kanovsky P, Ruzicka E, et al. Efficacy and safety of a standardised 500 unit dose of Dysport (Clostridium botulinum toxin type A haemaglutinin complex) in a heterogeneous cervical dystonia population: results of a prospective, multicentre, randomised, double-blind, placebo-controlled, parallel group study. J Neurol 2001;248:1073-8.

7 Dressler D. Clinical presentation and management of antibody-induced failure of botulinum toxin therapy. Mov Disord 2004;19:S92-S100.

8 Haussermann P, Marczoch S, Klinger C, et al. Long-term follow-up of cervical dystonia patients treated with botulinum toxin A. Mov Disord 2004;19:303-8.

9 Mohammadi B, Buhr N, Bigalke $\mathrm{H}$, et al. A long-term follow-up of botulinum toxin A in cervical dystonia. Neurol Res 2009;31:436-66.

10 Ferreira JJ, Colosimo C, Bhidayasiri R, et al. Factors influencing secondary non-response to botulinum toxin type A injections in cervical dystonia. Parkinsonism Relat Disord 2015;21:111-15.

11 Comella CL, Tanner CM, DeFoor-Hill L, et al. Dysphagia after botulinum toxin injections for spasmodic torticollis: clinical and radiologic findings. Neurology 1992;42:1307-10.

12 Bakheit AM, Liptrot A, Newton R, et al. The effect of total cumulative dose, number of treatment cycles, interval between injections, and length of treatment on the frequency of occurrence of antibodies to botulinum toxin type $\mathrm{A}$ in the treatment of muscle spasticity. Int J Rehabil Res 2012;35:36-9.

13 Truong DD, Gollomp SM, Jankovic J. Sustained efficacy and safety of repeated incobotulinum A (Xeomin) injections in blepharosapsm. J Neural Transm 2013;120:1345-53.

14 Park J, Lee MS, Harrison AR. Profile of Xeomin ${ }^{\circledR}$ (incobotulinumtoxinA) for the treatment of blepharospasm. Clin Ophthalmol 2011;5:725-32.

15 Jankovic J, Vuong KD, Ahsan J. Comparison of efficacy and immunogenicity of original versus current botulinum toxin in cervical dystonia. Neurology 2003;60:1186-8.

16 Brin MF, Comella CL, Jankovic J, et al. Long-term treatment with botulinum toxin type A in cervical dystonia has low immunogenicity by mouse protection assay. Mov Disord 2008;23:1353-60.

17 Ruiz PJ, Castrillo JC, Burguera JA, et al. Evolution of dose and response to botulinum toxin $\mathrm{A}$ in cervical dystonia: a multicenter study. J Neurol 2011;258:1055-7.

18 Chinnapongse RB, Lew MF, Ferreira JJ, et al. Immunogenicity and long-term efficacy of botulinum toxin type $\mathrm{B}$ in the treatment of cervical dystonia: report of 4 prospective, multicenter trials. Clin Neuropharmacol 2012;35:215-23.

19 Berman B, Seeberger L, Kumar R. Long-term safety, efficacy, dosing, and development of resistance with botulinum toxin type B in cervical dystonia. Mov Disord $2005 ; 20: 233-7$. 
20 Dressler D, Bigalke H, Benecke R. Botulinum toxin type B in antibody-induced botulinum toxin type A therapy failure. J Neurol 2003;250:967-9.

21 Hefter H, Hartmann C, Kahlen U, et al. Prospective analysis of neutralising antibody titres in secondary non-responders under continuous treatment with a botulinumtoxin type A preparation free of complexing proteins-a single cohort 4-year follow-up study. BMJ Open 2012;2.

22 Kamm C, Schümann F, Mix E, Benecke R. Secondary antibody-induced treatment failure under therapy with incobotulinumtoxinA $\left(\mathrm{Xeomin}{ }^{\circledR}\right)$ in a patient with segmental dystonia pretreated with abobotulinumtoxinA (Dysport ${ }^{\circledR}$ ). J Neurol Sci 2015;350:110-1.

23 Hefter H, Spiess C, Rosenthal D. Very early reduction in efficacy of botulinum toxin therapy for cervical dystonia in patients with subsequent secondary treatment failure: a retrospective analysis. J Neural Transm (Vienna) 2014;121:513-19.

24 Ferreira JJ, Bhidayasiri R, Colosimo C, et al. Survey of practices employed by neurologists for the definition and management of secondary non-response to botulinum toxin in cervical dystonia. Funct Neurol 2012;27:225-30.

25 Brin MF, Comella CL, Jankovic J, et al. Long-term treatment with botulinum toxin type A in cervical dystonia has low immunogenicity by mouse protection assay. Mov Disord 2008;23:1353-60.

26 Cordivari C, Misra VP, Vincent A, et al. Secondary nonresponsiveness to botulinum toxin $\mathrm{A}$ in cervical dystonia: the role of electromyogram-guided injections, botulinum toxin A antibody assay, and the extensor digitorum brevis test. Mov Disord 2006;21:1737-41.
27 Marsden CD, Marion MH, Quinn N. The treatment of severe dystonia in children and adults. J Neurol Neurosurg Psychiatr 1984;47:1166-73.

28 Brans JW, Lindeboom R, Snoek JW, et al. Botulinum toxin versus trihexyphenidyl in cervical dystonia: a prospective, randomized, double-blind controlled trial. Neurology 1996;46:1066-72.

29 Maltese M, Martella G, Madeo G, et al. Anticholinergic drugs rescue synaptic plasticity in DYT1 dystonia: role of M1 muscarinic receptors. Mov Disord 2014; 29:1655-65.

30 Gray SL, Anderson ML, Dublin S, et al. Cumulative use of strong anticholinergics and incident dementia: a prospective cohort study. Jama Intern Med 2015;175:401-7.

31 Volkmann J, Mueller J, Deuschl G, et al. Pallidal neurostimulation in patients with medication-refractory cervical dystonia: a randomised, sham-controlled trial. Lancet Neurol 2014;13:875-84.

32 Contarino MF, Van Den Munckhof P, Tijssen MA, et al. Selective peripheral denervation: comparison with pallidal stimulation and literature review. J Neurol 2014;261:300-8.

33 Huebl J, Brücke C, Schneider GH, et al. Bradykinesia induced by frequency-specific pallidal stimulation in patients with cervical and segmental dystonia. Parkinsonism Relat Disord 2015;21:800-3.

34 Flowers JM, Hicklin LA, Marion MH. Anterior and posterior sagittal shift in cervical dystonia: a clinical and electromyographic study, including a new EMG approach of the longus colli muscle. Mov Disord 2011;26:2409-14. 